Research Article

\title{
Classification of $\mathbf{f}$-Biharmonic Curves in Lorentz-Minkowski Space
}

\author{
Li Du (D) and Juan Zhang \\ School of Science, Chongqing University of Technology, Chongqing 400054, China \\ Correspondence should be addressed to Li Du; duli820210@cqut.edu.cn
}

Received 20 June 2020; Accepted 23 July 2020; Published 18 August 2020

Academic Editor: Antonio Masiello

Copyright (C) $2020 \mathrm{Li} \mathrm{Du}$ and Juan Zhang. This is an open access article distributed under the Creative Commons Attribution License, which permits unrestricted use, distribution, and reproduction in any medium, provided the original work is properly cited.

In this paper, we firstly derived the equations for the curves of a Lorentz-Minkowski space $\mathbb{L}^{3}$ to be $f$-biharmonic. Then, using these equations, we classify such unit speed curves in $\mathbb{L}^{3}$.

\section{Introduction}

Biharmonic isometric immersions are critical points of the bienergy functional (proposed by Eells and Lemaire in [1])

$$
E_{2}(\phi)=\frac{1}{2} \int_{M_{r}^{n}}\langle\tau(\phi), \tau(\phi)\rangle v_{g},
$$

for isometric immersions $\phi: M_{r}^{n} \longrightarrow N_{q}^{n+p}$ from an $n$-dimensional pseudo-Riemannian manifold $M_{r}^{n}$ into an $(n+p)$-dimensional pseudo-Riemannian manifold $N_{q}^{n+p}$, where $\tau(\phi)=\operatorname{tr} \nabla \mathrm{d} \phi=n \vec{H}$ (cf. [2,3]), with $\vec{H}$ be the mean curvature vector field of $M_{r}^{n}$, is the tension field of $\phi$ vanishing of which means that $\phi$ is harmonic or $M_{r}^{n}$ is minimal. The first variation formula for the bienergy $E_{2}(\phi)$ which is derived by Jiang in [4] shows that the Euler-Lagrange equation for $E_{2}(\phi)$ is

$$
\tau_{2}(\phi):=\operatorname{trace}\left(\nabla^{\phi} \nabla^{\phi}-\nabla_{\nabla}^{\phi}\right) \tau(\phi)-\operatorname{trace} \widetilde{R}(\mathrm{~d} \phi, \tau(\phi)) \mathrm{d} \phi=0,
$$

where $\widetilde{R}, \nabla^{\phi}$, and $\nabla$ are the curvature tensor of $N_{q}^{n+p}$, the induced connection by $\phi$ on the bundle $\phi^{-1} T N_{q}^{n+p}$, and the connection of $M_{r}^{n}$, respectively (cf. [4, 5] for $q=0$, and [3] for $q>0$, for details).

As a generalization of biharmonic isometric immersions, the $f$-biharmonic isometric immersion $\phi$ was introduced by
Lu in [6] (cf. [7] for $f$-biharmonic maps), as a critical point of the $f$-bienergy functional:

$$
E_{2, f}(\phi)=\frac{1}{2} \int_{M_{r}^{n}} f\langle\tau(\phi), \tau(\phi)\rangle v_{g},
$$

where $f$ is a fixed function $M_{r}^{n} \longrightarrow(0,+\infty)$.

The Euler-Lagrange equation gives the $f$-biharmonic isometric immersion (derived by $\mathrm{Lu}$ in [6])

$$
\tau_{2, f}(\phi):=f \tau_{2}(\phi)+(\Delta f) \tau(\phi)+2 \nabla_{\operatorname{grad} f}^{\phi} \tau(\phi)=0,
$$

where $\Delta$ is the laplace operator of $M_{r}^{n}$.

A submanifold is called a $f$-biharmonic submanifold if its isometric immersion $\phi$ is $f$-biharmonic (cf. [8]). When $f$ is a constant, $f$-biharmonic submanifolds are called biharmonic submanifolds (i.e., its bitension field $\tau_{2}(\phi)$ vanishes identically) (cf. [5]) which are called submanifolds with harmonic mean curvature vector field by Chen in [9].

The study of biharmonic submanifolds is a vibrant research subject, which was originated in $[4,5]$ by Jiang for his study of Euler-Lagrange's equation of the bienergy functional and also independently by Chen (cf. [10]) in his program of understanding the finite type submanifolds in Euclidean spaces, and there were numerous important developments in this domain over the past 40 plus years. For example, Dimitríc proved (cf. [11]) that any biharmonic 
curve in a Euclidean space is a geodesic (Chen and Ishikawa in [12] obtained the same result independently). Then, Caddeo, Montaldo, and Piu in [13] considered biharmonic curves on a surface and giave some examples of nongeodesic biharmonic curves. Later, Caddeo, Montaldo, and Oniciuc (cf. [2]) showed nonexistence of nongeodesic biharmonic curves in a 3-dimensional hyperbolic space and proved that nongeodesic biharmonic curves in the unit 3-sphere are circles of geodesic curvature 1 or helices which are geodesics in the Clifford minimal torus. Also, Chen and Ishikawa (cf. $[12,14,15])$ classified completely unit speed biharmonic curves in pseudo-Euclidean spaces $\mathbb{E}_{q}^{3}(q=0,1,2,3)$ (when $q=0, \mathbb{E}_{0}^{3}$ is the Euclidean space $\left.\mathbb{E}^{3}\right)$ and gave some examples of nonminimal biharmonic curves. More generally, Sasahara in [3] considered unit biharmonic curves in nonflat Lorentz 3-space forms and obtained full classification of such curves. For the study of biharmonic curves in other model spaces, we refer to [16-19] with references therein. For some recent progress of biharmonic submanifolds (instead of biharmonic curves), we refer readers to $[2,5,12-14,16,17,20-24]$ and the references therein.

Naturally, the next step has been the study of $f$-biharmonic curves. Ou in [8] derived equations for $f$-biharmonic curves in a generic manifold and completely classified $f$-biharmonic curves in 3-dimensional Euclidean space $\mathbb{E}^{3}$, where he proved that such curves in $\mathbb{E}^{3}$ are planar curves or general helices and gave some examples of nonbiharmonic $f$-biharmonic curves in $\mathbb{E}^{3}$. After that, there are a few valuable results on $f$-biharmonic curves in (generalized) Sasakian space forms, Sol spaces, Cartan-Vranceanu 3-dimensional spaces, or homogeneous contact 3-manifolds; we refer to [25-27].

These facts motivate us to study $f$-biharmonic curves in pseudo-Riemannian manifolds since it helps to bridge the gap between modern differential geometry and the mathematical physics of general relativity. In this paper, we will investigate unit speed $f$-biharmonic curves with a positive function $f$ in Lorentz-Minkowski space $\mathbb{L}^{3}$ and obtain the following classification theorems.

Theorem 1. A curve $\gamma:(a, b) \longrightarrow \mathbb{L}^{3}$ parametrized by arclength $s$ is an $f$-biharmonic Frenet curve if and only if one of the following cases holds:

(i) $\gamma$ is a circular helix and $f$ is constant, and $\gamma=1 / 6\left(\kappa \tau s^{3},-\kappa^{2} s^{3}+6 s, 3 \kappa s^{2}\right)$ for timelike binormal, $\gamma=1 / 6\left(\kappa^{2} s^{3}+6 s, 3 \kappa s^{2}, \kappa \tau s^{3}\right)$ for timelike tangent

(ii) $\gamma$ is a planar curve and $f=c_{1} \kappa^{-3 / 2}$ with $\tau=0$ and $\kappa=4 c_{2} / c_{2}^{2}\left(s+c_{3}\right)^{2}-16$ for spacelike binormal or $\kappa=4 c_{2} / c_{2}^{2}\left(s+c_{3}\right)^{2}+16$ for timelike binormal

(iii) $\gamma$ is a helix and $f=c_{1} \kappa^{-3 / 2}$ with $\tau=c \kappa$ and $\kappa=$ $4 c_{2} / c_{2}^{2}\left(s+c_{3}\right)^{2}+16\left(1-c^{2}\right)$ for timelike binormal, or $\kappa=4 c_{2} / c_{2}^{2}\left(s+c_{3}\right)^{2}-16\left(1+c^{2}\right)$ for timelike principal normal, or $\kappa=4 c_{2} / c_{2}^{2}\left(s+c_{3}\right)^{2}+16\left(c^{2}-1\right)$ for timelike tangent

where $\kappa>0$ and $\tau$ are the curvature and torsion of $\gamma$.
Theorem 2. A curve $\gamma:(a, b) \longrightarrow \mathbb{L}^{3}$ parametrized by arclength $s$ is an $f$-biharmonic unit speed curve with lightlike principal normal if and only if one of the following cases holds:

(i) $\gamma$ is a planar curve with $\tilde{\tau}=0$ and $f=c_{1} s+c_{2}$, and $\gamma=\left(s^{2} / 2, s, s^{2} / 2\right)$

(ii) $\gamma$ is a helix curve and $f=\left(c_{1}+c_{2} s\right) e^{-\tilde{\tau} s}$ with $\tilde{\tau}$ being nonzero constant, and $\gamma=1 / \tilde{\tau}^{2}(\cosh (\tilde{\tau} s)+$ $\left.\sinh (\tilde{\tau} s), \tilde{\tau}^{2} s, \cosh (\widetilde{\tau} s)+\sinh (\widetilde{\tau} s)\right)$

(iii) $\gamma$ is a helix curve and $f=\left(c_{1}+c_{2} s\right) e^{-\int_{\tau}^{-d s}}$ with $\tilde{\tau}$ being nonconstant

where $\tilde{\tau}$ is the torsion of $\gamma, c_{1}$, and $c_{2}$ are two constants.

\section{Preliminaries}

Let $\mathbb{E}_{1}^{3}$ be a pseudo-Euclidean 3-space with metric given by

$$
\langle,\rangle=-\mathrm{d} x_{1}^{2}+\mathrm{d} x_{2}^{2}+\mathrm{d} x_{3}^{2},
$$

where $\left(x_{1}, x_{2}, x_{3}\right)$ is the natural co-ordinate system of $\mathbb{E}_{1}^{3}$. The $\mathbb{E}_{1}^{3}$ is also called 3-dimensional Lorentz-Minkowski space, denoted by $\mathbb{L}^{3}$. Since $\langle$,$\rangle is an indefinite metric, an$ arbitrary vector $v \in \mathbb{L}^{3}$ can have one of three Lorentz causal characters: it can be spacelike if $\langle v, v\rangle>0$ or $v=0$, timelike if $\langle v, v\rangle\langle 0$ and lightlike or null if $\langle v, v\rangle=0$ or $v \neq 0$.

Let $\gamma:(a, b) \longrightarrow \mathbb{L}^{3}$ be an arbitrary curve in $\mathbb{L}^{3}$ and $\gamma$ can have locally one of the following causal characters: $\gamma$ is spacelike, lightlike (null), or timelike, if $\left\langle\gamma^{\prime}, \gamma^{\prime}\right\rangle$ is bigger, equal or smaller than 0 on an interval $(a, b)$.

When $\gamma$ is non-lightlike, then $\gamma$ is parametrized by arc length $s$. Specifying that if a spacelike (resp. timelike) curve is parametrized by arc length, then the velocity vector satisfies respectively $\left\langle\gamma^{\prime}, \gamma^{\prime}\right\rangle=1$, (resp. $\left\langle\gamma^{\prime}, \gamma^{\prime}\right\rangle=-1$ ).

A curve $\gamma:(a, b) \longrightarrow \mathbb{L}^{3}$ is said to be a unit speed curve if the velocity vector field of $\gamma^{\prime}$ satisfies

$$
\left\langle\gamma^{\prime}, \gamma^{\prime}\right\rangle=\varepsilon_{1}= \pm 1
$$

Differentiating (6), we have

$$
\left\langle\gamma^{\prime \prime}, \gamma^{\prime}\right\rangle=0
$$

In general, the causal character of $\gamma^{\prime \prime}$ may change in the interval $(a, b)$, but the continuity assures that $\gamma^{\prime \prime}$ has the same spacelike, timelike, or lightlike in an interval around $p$; we refer the readers to (examples, pp: 15-16) [28]. Thus, we will assume that the causal character of $\gamma^{\prime}$ or $\gamma^{\prime \prime}$ is the same in $(a, b)$. Also, we have from (7) that $\gamma^{\prime \prime}$ is perpendicular to $\gamma^{\prime}$ or the curvature of $\gamma$ is zero identically (i.e., $\gamma$ is a straightline). In the following, we will give the Frenet formulas of $\gamma$ with nonzero curvature depending on $\left\langle\gamma^{\prime \prime}, \gamma^{\prime \prime}\right\rangle$.

When $\gamma$ is a unit speed curve with $\left\langle\gamma^{\prime \prime}, \gamma^{\prime \prime}\right\rangle \neq 0, \gamma$ is called a Frenet curve in $\mathbb{L}^{3}$. Every Frenet curve $\gamma$ in $\mathbb{L}^{3}$ admits a Frenet frame field along $\gamma$. Here, a Frenet frame field $P=$ $\left(\mathbf{p}_{1}, \mathbf{p}_{2}, \mathbf{p}_{3}\right)$ is an orthonormal frame field along $\gamma$ such that $\mathbf{p}_{1}=\gamma^{\prime}$, with $\mathbf{p}_{2}$ being parallel to $\gamma^{\prime \prime}$ and $\mathbf{p}_{3}$ being perpendicular to the plane $\left\{\mathbf{p}_{1}, \mathbf{p}_{2}\right\}$. We call $\mathbf{p}_{1}, \mathbf{p}_{2}$, and $\mathbf{p}_{3}$ the tangent vector field, principal normal vector field, and binormal vector field of $\gamma$, respectively, and $\left\{\mathbf{p}_{1}, \mathbf{p}_{2}, \mathbf{p}_{3}\right\}$ satisfies the following Frenet formula. 
Lemma 1. Let $\gamma:(a, b) \longrightarrow \mathbb{L}^{3}$ be a Frenet curve with arclength parameter in $\mathbb{L}^{3}$, then the Frenet formulas of $\gamma$ are, in matrixflotation:

$$
\nabla_{\gamma^{\prime}}^{\mathbb{L}^{3}} P=\left(\begin{array}{ccc}
0 & \kappa & 0 \\
-\varepsilon_{1} \varepsilon_{2} \kappa & 0 & -\varepsilon_{2} \varepsilon_{3} \tau \\
0 & \tau & 0
\end{array}\right)\left(\begin{array}{l}
\mathbf{p}_{1} \\
\mathbf{p}_{2} \\
\mathbf{p}_{3}
\end{array}\right),
$$

where the functions $\kappa(>0)$ and $\tau$ are called the curvature and torsion of $\gamma$, respectively, and

$$
\begin{aligned}
& \left\langle\mathbf{p}_{i}, \mathbf{p}_{i}\right\rangle=\varepsilon_{i}, \quad i=1,2,3, \\
& \left\langle\mathbf{p}_{i}, \mathbf{p}_{j}\right\rangle=0, \quad i, j=1,2,3, i \neq j .
\end{aligned}
$$

Proof. We set

$$
\nabla_{\gamma^{\prime}}^{\mathbb{L}^{3}} \mathbf{p}_{1}=\kappa \mathbf{p}_{2}
$$

where $\kappa(>0)$ is the curvature of $\gamma$. Note that

$$
\begin{aligned}
& \left\langle\mathbf{p}_{3}, \mathbf{p}_{1}\right\rangle=0, \\
& \left\langle\mathbf{p}_{3}, \mathbf{p}_{3}\right\rangle=\varepsilon_{3} .
\end{aligned}
$$

Then, differentiating the above equation, combining with (10), we get

$$
\begin{aligned}
& \nabla_{\gamma^{\prime}}^{\mathbb{Z}^{3}} \mathbf{p}_{3} \perp \mathbf{p}_{1}, \\
& \nabla_{\gamma^{\prime}}^{\mathbb{3}^{3}} \mathbf{p}_{3} \perp \mathbf{p}_{3},
\end{aligned}
$$

which mean that $\nabla_{\gamma^{\prime}}^{\mathbb{L}^{3}} \mathbf{p}_{3}$ is parallel to $\mathbf{p}_{2}$, i.e.,

$$
\nabla_{\gamma^{\prime}}^{\mathbb{L}^{3}} \mathbf{p}_{3}=\tau \mathbf{p}_{2}
$$

where $\tau$ is the torsion of $\gamma$.

On the other hand, it is easy to see that the vector $\nabla_{v^{\prime}}^{\mathbb{L}^{3}} \mathbf{p}_{2} \in \mathbb{L}^{3}$, then there exist three functions $f_{1}, f_{2}$, and $f_{3}$, such that

$$
\nabla_{\gamma^{\prime}}^{\mathbb{L}^{3}} \mathbf{p}_{2}=f_{1} \mathbf{p}_{1}+f_{2} \mathbf{p}_{2}+f_{3} \mathbf{p}_{3}
$$

Taking the scalar product with $\mathbf{p}_{1}, \mathbf{p}_{2}$, and $\mathbf{p}_{3}$, respectively, we obtain

$$
\begin{aligned}
& \left\langle\nabla_{\dot{\gamma}}^{\mathbb{Q}^{3}} \mathbf{p}_{2}, \mathbf{p}_{1}\right\rangle=\varepsilon_{1} f_{1}, \\
& \left\langle\nabla_{\dot{\gamma}}^{\mathbb{Q}^{3}} \mathbf{p}_{2}, \mathbf{p}_{2}\right\rangle=\varepsilon_{2} f_{2}, \\
& \left\langle\nabla_{\dot{\gamma}}^{\mathbb{Q}^{3}} \mathbf{p}_{2}, \mathbf{p}_{3}\right\rangle=\varepsilon_{3} f_{3} .
\end{aligned}
$$

Also, differentiating both of the following equations:

$$
\begin{aligned}
& \left\langle\mathbf{p}_{2}, \mathbf{p}_{2}\right\rangle=\varepsilon_{2}, \\
& \left\langle\mathbf{p}_{2}, \mathbf{p}_{1}\right\rangle=0, \\
& \left\langle\mathbf{p}_{2}, \mathbf{p}_{3}\right\rangle=0,
\end{aligned}
$$

using (10) and (13), we have

$$
\begin{aligned}
& \left\langle\nabla_{\gamma}^{\mathbb{Q}^{3}} \mathbf{p}_{2}, \mathbf{p}_{2}\right\rangle=0, \\
& \left\langle\nabla_{\gamma}^{\mathbb{Q}^{3}} \mathbf{p}_{2}, \mathbf{p}_{1}\right\rangle=-\kappa \varepsilon_{2}, \\
& \left\langle\nabla_{\gamma}^{\mathbb{L}^{3}} \mathbf{p}_{2}, \mathbf{p}_{3}\right\rangle=-\tau \varepsilon_{2} .
\end{aligned}
$$

Together with (15) leads to

$$
\begin{aligned}
& f_{1}=-\varepsilon_{1} \varepsilon_{2} \kappa, \\
& f_{2}=0, \\
& f_{3}=-\varepsilon_{2} \varepsilon_{3} \tau .
\end{aligned}
$$

Substituting into (14), and completing the proof of Lemma 1.

Remark 1. The Frenet formula 8 has appeared in [29-31] in different forms, but the detail proof of (8) is not given in those papers. Thus, we give a brief proof of (8) for completeness and simplicity of our main results.

When $\gamma$ is a unit speed curve with $\left\langle\gamma^{\prime \prime}, \gamma^{\prime \prime}\right\rangle=0$, then we choose a suitable pseudo-orthonormal frame field $\widetilde{P}=\left(\widetilde{\mathbf{p}}_{1}, \widetilde{\mathbf{p}}_{2}, \widetilde{\mathbf{p}}_{3}\right)$ along $\gamma$ in $\mathbb{L}^{3}$ with $\widetilde{\mathbf{p}}_{1}, \widetilde{\mathbf{p}}_{2}$, and $\widetilde{\mathbf{p}}_{3}$ being tangent vector field, principal normal vector field, and binormal vector field of $\gamma$, respectively, such that (cf. [32])

$$
\nabla_{\gamma}^{\mathbb{Q} 3} \widetilde{P}=\left(\begin{array}{ccc}
0 & \widetilde{\kappa} & 0 \\
0 & \widetilde{\tau} & 0 \\
-\widetilde{\kappa} & 0 & \widetilde{\tau}
\end{array}\right)\left(\begin{array}{c}
\widetilde{\mathbf{p}}_{1} \\
\widetilde{\mathbf{p}}_{2} \\
\widetilde{\mathbf{p}}_{3}
\end{array}\right),
$$

where the functions $\widetilde{\mathcal{\kappa}}(\geq 0)$ and $\widetilde{\tau}$ are called the curvature and torsion, respectively, and

$$
\begin{aligned}
& \left\langle\widetilde{\mathbf{p}}_{1}, \widetilde{\mathbf{p}}_{1}\right\rangle=1, \\
& \left\langle\widetilde{\mathbf{p}}_{2}, \widetilde{\mathbf{p}}_{2}\right\rangle=\left\langle\widetilde{\mathbf{p}}_{3}, \widetilde{\mathbf{p}}_{3}\right\rangle=0, \\
& \left\langle\widetilde{\mathbf{p}}_{2}, \widetilde{\mathbf{p}}_{3}\right\rangle=1, \\
& \left\langle\widetilde{\mathbf{p}}_{1}, \widetilde{\mathbf{p}}_{2}\right\rangle=\left\langle\widetilde{\mathbf{p}}_{1}, \widetilde{\mathbf{p}}_{3}\right\rangle=0 .
\end{aligned}
$$

In this case, the curvature $\widetilde{\kappa}$ can take only two values: $\widetilde{\kappa}=0$ when $\gamma$ is a straight line; $\widetilde{\kappa}=1$ in all other cases. Thus, we have from (19) that $\left\{\widetilde{\mathbf{p}}_{1}, \widetilde{\mathbf{p}}_{2}, \widetilde{\mathbf{p}}_{3}\right\}$ satisfies the following Frenet formula in matrixflotation:

$$
\nabla_{\dot{\gamma}}^{\mathbb{U}^{3}} \widetilde{P}=\left(\begin{array}{ccc}
0 & 1 & 0 \\
0 & \widetilde{\tau} & 0 \\
-1 & 0 & \widetilde{\tau}
\end{array}\right)\left(\begin{array}{c}
\widetilde{\mathbf{p}}_{1} \\
\widetilde{\mathbf{p}}_{2} \\
\widetilde{\mathbf{p}}_{3}
\end{array}\right) .
$$

Next, we derived the equations for the unit speed curve $\gamma$ to be $f$-biharmonic.

Lemma 2. A curve $\gamma:(a, b) \longrightarrow \mathbb{L}^{3}$ parametrized by arclength $s$ is an $f$-biharmonic unit speed curve if and only if

$$
f\left(\nabla_{\gamma^{\prime}}^{\mathbb{L}^{3}} \nabla_{\gamma^{\prime}}^{\mathbb{L}^{3}} \nabla_{\gamma^{\prime}}^{\mathbb{L}^{3}} \gamma^{\prime}\right)+2 f \prime \nabla_{\gamma^{\prime}}^{\mathbb{L}^{3}} \nabla_{\gamma^{\prime}}^{\mathbb{L}^{3}} \gamma^{\prime}+f^{\prime \prime} \nabla_{\gamma^{\prime}}^{\mathbb{L}^{3}} \gamma^{\prime}=0 .
$$

Proof. Let $\gamma=\gamma(s)$ be parametrized by arclength $s$. Then $e_{1}=\partial / \partial s$ is an orthonormal frame on $((a, b), \mathrm{d} s)$ and 


$$
\mathrm{d} \gamma\left(e_{1}\right)=\mathrm{d} \gamma\left(\frac{\partial}{\partial s}\right)=\gamma^{\prime} .
$$

Then the tension field of $\gamma$ is given by

$$
\tau(\gamma)=\varepsilon_{1} \nabla_{e_{1}}^{\gamma} \mathrm{d} \gamma\left(e_{1}\right)=\varepsilon_{1} \nabla_{\gamma^{\prime}}^{\mathbb{L}^{3}} \gamma^{\prime}
$$

For a function $f:(a, b) \longrightarrow(0, \infty)$, we have

$$
\operatorname{grad} f=\varepsilon_{1} f^{\prime} e_{1}, \quad \Delta f=\varepsilon_{1} f^{\prime \prime} .
$$

A straightforward computation gives

$$
\nabla_{\operatorname{grad}}^{\gamma} \tau(\gamma)=\varepsilon_{1} f^{\prime} \nabla_{e_{1}}^{\gamma} \tau(\gamma)=\varepsilon_{1} f^{\prime} \nabla_{\gamma^{\prime}}^{\mathbb{L}^{3}}\left(\varepsilon_{1} \nabla_{\gamma^{\prime}}^{\mathbb{L}^{3}} \gamma^{\prime}\right)=f^{\prime} \nabla_{\gamma^{\prime}}^{\mathbb{L}^{3}} \nabla_{\gamma^{\prime}}^{\mathbb{L}^{3}} \gamma^{\prime},
$$

$$
(\Delta f) \tau(\gamma)=f^{\prime \prime} \nabla_{\gamma^{\prime}}^{\mathbb{Q}^{3}} \gamma^{\prime} .
$$

Choose a normal coordinates at a point in $(a, b)$; it follows from (24) that

$$
\tau_{2}(\gamma)=\varepsilon_{1} \nabla_{e_{1}}^{\gamma} \nabla_{e_{1}}^{\gamma} \tau(\gamma)=\nabla_{\gamma^{\prime}}^{\mathbb{L}^{3}} \nabla_{\gamma^{\prime}}^{\mathbb{L}^{3}} \nabla_{\gamma^{\prime}}^{\mathbb{L}^{3}} \gamma^{\prime} .
$$

Putting (26)-(28) into (4), we obtain that equation (22) holds and Lemma 2 follows.

Finally, we will give several definitions of curves in $\mathbb{L}^{3}$.

A helix $\gamma:(a, b) \longrightarrow \mathbb{L}^{3}$ is a curve parametrized by arclength such that there exists a vector $v \in \mathbb{L}^{3}$ with the property that the function $\left\langle\gamma^{\prime}, v\right\rangle$ is constant. Then the curve $\gamma$ is a helix if and only if the ratio of the curvature and torsion of $\gamma$ is a constant. If both the curvature (nonzero) and the torsion of $\gamma$ are constant, then the $\gamma$ curve is called a circular helix (cf. [28]).

A curve $\gamma:(a, b) \longrightarrow \mathbb{L}^{3}$ is called a planar curve if the torsion identically vanishes (cf. [32]).

It is obvious to see that a straight-line (i.e., the curvature identically vanishes) and a planar curve are helices.

\section{Main Theorems and Their Proofs}

Proof of Theorem 1. We have from (8) that

$$
\left\{\begin{array}{l}
\nabla_{\dot{\gamma}}^{\mathbb{Q}^{3}} \mathbf{p}_{1}=\kappa \mathbf{p}_{2}, \\
\nabla_{\gamma}^{\mathbb{Q}^{3}} \mathbf{p}_{2}=\left(-\varepsilon_{1} \varepsilon_{2}\right) \kappa \mathbf{p}_{1}+\left(-\varepsilon_{2} \varepsilon_{3}\right) \tau \mathbf{p}_{3}, \\
\nabla_{\gamma}^{\mathbb{L}^{3}} \mathbf{p}_{3}=\tau \mathbf{p}_{3} .
\end{array}\right.
$$

Now, taking into account the first and second equation of (29), we obtain

$$
\begin{aligned}
\nabla_{\dot{\gamma}}^{\mathbb{L}^{3}} \nabla_{\dot{\gamma}}^{\mathbb{L}^{3}} \gamma^{\prime} & =\kappa^{\prime} \mathbf{p}_{2}+\kappa \nabla_{\dot{\gamma}}^{\mathbb{L}^{3}} \mathbf{p}_{2} \\
& =\left(-\varepsilon_{1} \varepsilon_{2}\right) \kappa^{2} \mathbf{p}_{1}+\kappa^{\prime} \mathbf{p}_{2}+\left(-\varepsilon_{2} \varepsilon_{3}\right) \kappa \tau \mathbf{p}_{3},
\end{aligned}
$$

which together with (29) shows that

$$
\begin{aligned}
\nabla_{\gamma^{\prime}}^{\mathbb{L}^{3}} \nabla_{\gamma^{\prime}}^{\mathbb{L}^{3}} \nabla_{\gamma^{\prime}}^{\mathbb{L}^{3}} \gamma^{\prime}= & \nabla_{\gamma^{\prime}}^{\mathbb{L}^{3}}\left(\left(-\varepsilon_{1} \varepsilon_{2}\right) \kappa^{2} \mathbf{p}_{1}+\kappa^{\prime} \mathbf{p}_{2}+\left(-\varepsilon_{2} \varepsilon_{3}\right) \kappa \tau \mathbf{p}_{3}\right) \\
= & 2\left(-\varepsilon_{1} \varepsilon_{2}\right) \kappa \kappa^{\prime} \mathbf{p}_{1}+\left(-\varepsilon_{1} \varepsilon_{2}\right) \kappa^{2} \nabla_{\gamma^{\prime}}^{\mathbb{L}^{3}} \mathbf{p}_{1}+\kappa^{\prime \prime} \mathbf{p}_{2} \\
& +\kappa^{\prime} \nabla_{\gamma^{\prime}}^{\mathbb{L}^{3}} \mathbf{p}_{2}+\left(-\varepsilon_{2} \varepsilon_{3}\right) \kappa^{\prime} \tau \mathbf{p}_{3}+\left(-\varepsilon_{2} \varepsilon_{3}\right) \kappa \tau^{\prime} \mathbf{p}_{3} \\
& +\left(-\varepsilon_{2} \varepsilon_{3}\right) \kappa \tau \nabla_{\gamma^{\prime}}^{\mathbb{L}^{3}} \mathbf{p}_{3} \\
= & 3\left(-\varepsilon_{1} \varepsilon_{2}\right) \kappa \kappa^{\prime} \mathbf{p}_{1} \\
& +\left(\left(-\varepsilon_{1} \varepsilon_{2}\right) \kappa^{3}+\kappa^{\prime \prime}+\left(-\varepsilon_{1} \varepsilon_{2}\right) \kappa \tau^{2}\right) \mathbf{p}_{2} \\
& +\left(2\left(-\varepsilon_{2} \varepsilon_{3}\right) \kappa \prime \tau+\left(-\varepsilon_{2} \varepsilon_{3}\right) \kappa \tau^{\prime}\right) \mathbf{p}_{3} .
\end{aligned}
$$

Putting the first equation of (29)-(31) into (22), we get that $\gamma$ is $f$-biharmonic if and only if

$$
\left\{\begin{array}{l}
2 \kappa^{2} f^{\prime}+3 \kappa \kappa^{\prime} f=0 \\
\kappa f^{\prime \prime}+2 \kappa^{\prime} f^{\prime}+\left(\left(-\varepsilon_{1} \varepsilon_{2}\right) \kappa^{3}+\kappa^{\prime \prime}+\left(-\varepsilon_{2} \varepsilon_{3}\right) \kappa \tau^{2}\right) f=0 \\
2 \kappa \tau f^{\prime}+2 \kappa^{\prime} \tau f+\tau^{\prime} \kappa f=0 .
\end{array}\right.
$$

In the following, we will investigate the characteristics of curves according to different values of $\kappa$ and $\tau$.

Case 1: When $\kappa$ is a nonzero constant, then it follows from the first equation of (32) that

$$
f^{\prime}=0
$$

It is obvious to see that $f$ is a constant, which implies that $\gamma$ is a biharmonic curve. Together with the third equation of (32), we obtain that $\tau$ is a constant. Hence the second equation of (32) can be simplified to

$$
\tau^{2}=-\varepsilon_{1} \varepsilon_{3} \kappa^{2}(\text { a nonzero constant })
$$

which means that $\gamma$ is a circular helix (cf. [28, 32]). We note that $\left(\varepsilon_{1}, \varepsilon_{3}\right)$ evaluates among the following two possible cases:

$$
\begin{aligned}
& (1,-1), \\
& (-1,1) .
\end{aligned}
$$

(i) When $\varepsilon_{1}=1$ and $\varepsilon_{3}=-1$, i.e., $\mathbf{p}_{1}$ is spacelike tangent and $\mathbf{p}_{3}$ is timelike binormal, then we conclude from Theorem 3.3 in [32] that

$$
\gamma(s)=\frac{1}{6}\left(\kappa \tau s^{3},-\kappa^{2} s^{3}+6 s, 3 \kappa s^{2}\right) .
$$


(ii) When $\varepsilon_{1}=-1$ and $\varepsilon_{3}=1$, i.e., $\mathbf{p}_{1}$ is timelike tangent and $\mathbf{p}_{3}$ is spacelike binormal, then we conclude from Theorem 3.6 in [32] that

$$
\gamma(s)=\frac{1}{6}\left(\kappa^{2} s^{3}+6 s, 3 \kappa s^{2}, \kappa \tau s^{3}\right)
$$

Conversely, according to the proof of Case 4 in Theorem 3.3 for (36) and Case 6 in Theorem 3.6 for (37), respectively, we know that their curvature $\kappa$ and torsion $\tau$ are two constants which satisfy $\kappa=\tau$. Combining with $f$ being a constant, we prove that equation (32) holds for the corresponding curves; that is, $\gamma$ is $f$-biharmonic.

Case 2: When $\tau$ is zero, then equation (32) is equivalent to

$$
\left\{\begin{array}{l}
2 \kappa^{2} f^{\prime}+3 \kappa \kappa^{\prime} f=0, \\
\kappa f^{\prime \prime}+2 \kappa^{\prime} f^{\prime}+\left(-\varepsilon_{1} \varepsilon_{2}\right) \kappa^{3} f+\kappa^{\prime \prime} f=0 .
\end{array}\right.
$$

Furthermore, we have

$$
\begin{aligned}
\frac{2 f^{\prime}}{f}+\frac{3 \kappa^{\prime}}{\kappa} & =0, \\
\frac{\kappa^{\prime \prime}}{\kappa}+2 \frac{\kappa^{\prime}}{\kappa} \frac{f^{\prime}}{f}+\frac{f^{\prime \prime}}{f}+\left(-\varepsilon_{1} \varepsilon_{2}\right) \kappa^{2} & =0 .
\end{aligned}
$$

A direct derivative calculation for $f^{2} \kappa^{3}$ gives

$$
\left(f^{2} \kappa^{3}\right)^{\prime}=f \kappa\left(2 \kappa^{2} f^{\prime}+3 \kappa \kappa^{\prime} f\right)
$$

then it follows from the first equation of (38) that $f^{2} \kappa^{3}=c$ with $c$ being a constant, which shows that

$$
f=c \kappa^{-3 / 2} \text {. }
$$

Also, differentiating both sides (39) yields

$$
\frac{f^{\prime \prime}}{f}=-\frac{3}{2} \frac{\kappa^{\prime \prime}}{\kappa}+\frac{15}{4} \frac{\left(\kappa^{\prime}\right)^{2}}{\kappa^{2}} .
$$

Putting (39) and (43) into (40), we deduce that

$$
3 \kappa^{\prime 2}-2 \kappa^{\prime \prime} \kappa-4\left(\varepsilon_{1} \varepsilon_{2}\right) \kappa^{4}=0 .
$$

Since $\varepsilon_{1} \varepsilon_{2}=-\varepsilon_{3}$, we have from (44) that, for spacelike binormal vector field,

$$
3 \kappa^{\prime 2}-2 \kappa^{\prime \prime} \kappa+4 \kappa^{4}=0
$$

and for timelike binormal vector field,

$$
3 \kappa^{\prime 2}-2 \kappa^{\prime \prime} \kappa-4 \kappa^{4}=0 .
$$

Solving the ODEs (45) and (46), respectively, we have that, for (45),

$$
\kappa=\frac{4 c_{2}}{c_{2}^{2} s^{2}+2 c_{2}^{2} c_{3} s+\left(c_{2} c_{3}\right)^{2}-16},
$$

and for (46),

$$
\kappa=\frac{4 c_{2}}{16+c_{2}^{2} s^{2}+2 c_{2}^{2} c_{3} s+\left(c_{2} c_{3}\right)^{2}} .
$$

Note that $\tau=0$; then $\gamma$ is a planar curve. Thus, the curve $\gamma$ is a planar curve with spacelike binormal and $\kappa=4 c_{2} / c_{2}^{2} s^{2}+2 c_{2}^{2} c_{3} s+\left(c_{2} c_{3}\right)^{2}-16$, or timelike binormal and $\kappa=4 c_{2} / 16+c_{2}^{2} s^{2}+2 c_{2}^{2} c_{3} s+\left(c_{2} c_{3}\right)^{2}$ and $f=c \kappa^{-3 / 2}$.

Conversely, because $f=c \kappa^{-3 / 2}$, it is not difficult to check that the first equation in (38) holds. Also, combining with $f=c \kappa^{-3 / 2}$, a long calculation for $\kappa=4 c_{2} / c_{2}^{2} s^{2}+2 c_{2}^{2} c_{3} s+$ $\left(c_{2} c_{3}\right)^{2}-16$ for spacelike binormal, or $\kappa=4 c_{2} / 16+c_{2}^{2} s^{2}+$ $2 c_{2}^{2} c_{3} s+\left(c_{2} c_{3}\right)^{2}$ for timelike binormal, respectively, proves that the second equation in (38) holds, that is, $\gamma$ is $f$-biharmonic.

Case 3: When $\tau$ is a nonzero constant, then equation (32) is equivalent to

$\left\{\begin{array}{l}\text { (i) } 2 \kappa^{2} f^{\prime}+3 \kappa^{\prime} \kappa f=0, \\ \text { (ii) } f^{\prime \prime} f+2 \kappa^{\prime} f^{\prime}+\left(\left(-\varepsilon_{1} \varepsilon_{2}\right) \kappa^{3}+\kappa^{\prime \prime}+\left(-\varepsilon_{2} \varepsilon_{3}\right) \kappa \tau^{2}\right) f=0, \\ \text { (iii) } \kappa f \prime+\kappa \prime f=0 .\end{array}\right.$

Combining with (i) and (iii) in (49), it is straightforward to prove that $f$ and $\kappa$ are two constants. Substituting those facts into (ii) in (49), we obtain that

$$
\kappa^{2}=\varepsilon_{2} \tau^{2}
$$

which implies that $\kappa$ is also a nonzero constant. Since $\varepsilon_{2}=-\varepsilon_{1} \varepsilon_{3}$, following the similar process as in Case 1, we complete the case.

Case 4: When $\kappa \neq$ constant and $\tau \neq$ constant, then equation (32) is equivalent to

$$
\left\{\begin{array}{l}
\text { (i) } f^{2} \kappa^{3}=c_{1}, \\
\text { (ii) } f^{\prime \prime} f+2 \kappa^{\prime} f^{\prime}+\kappa^{\prime \prime} f=\left(\left(\varepsilon_{1} \varepsilon_{2}\right) \kappa^{2}+\left(\varepsilon_{2} \varepsilon_{3}\right) \tau^{2}\right) \kappa f, \\
\text { (iii) } \kappa^{2} \tau f^{2}=\widetilde{c}_{2},
\end{array}\right.
$$

where $c_{1}$ and $\widetilde{c}_{2}$ are two constants. On one hand, by the first equation in (51), it is obvious to see that

$$
f=c_{1} \kappa^{-3 / 2}
$$

where $c_{1}>0$. Moreover, it follows from (i) and (iii) in (51) that $\tau / \kappa=c$ with $c$ being constant, then we conclude from [28] that $\gamma$ is a helix. 
On the other hand, combining with (43) and (i) and (ii) in (51), with much more tedious computations gives

$$
3 \kappa^{\prime 2}-2 \kappa^{\prime \prime} \kappa-4\left(\varepsilon_{1} \varepsilon_{2}+\varepsilon_{2} \varepsilon_{3} c^{2}\right) \kappa^{4}=0 .
$$

Note that $\left(\varepsilon_{1}, \varepsilon_{2}, \varepsilon_{3}\right)$ evaluates among the following three possible cases:

$$
\begin{aligned}
& (1,1,-1), \\
& (1,-1,1), \\
& (-1,1,1) .
\end{aligned}
$$

(i) When $\varepsilon_{1}=1, \varepsilon_{2}=1, \varepsilon_{3}=-1$, equation (53) becomes

$$
3 \kappa^{\prime 2}-2 \kappa^{\prime \prime} \kappa-4\left(1-c^{2}\right) \kappa^{4}=0
$$

Solving ODE (55), we have $\kappa=4 c_{2} / 16\left(1-c^{2}\right)+c_{2}^{2} s^{2}+2 c_{2}^{2} c_{3} s+\left(c_{2} c_{3}\right)^{2}$.

(ii) When $\varepsilon_{1}=1, \varepsilon_{2}=-1, \varepsilon_{3}=1$, equation (53) becomes

$$
3 \kappa^{\prime 2}-2 \kappa \prime \prime \kappa+4\left(1+c^{2}\right) \kappa^{4}=0
$$

Solving ODE (56), we have $\kappa=4 c_{2} / c_{2}^{2} s^{2}+2 c_{2}^{2} c_{3} s+\left(c_{2} c_{3}\right)^{2}-16\left(1+c^{2}\right)$.

(iii) When $\varepsilon_{1}=-1, \varepsilon_{2}=1, \varepsilon_{3}=1$, equation (53) becomes

$$
3 \kappa^{\prime 2}-2 \kappa^{\prime \prime} \kappa-4\left(c^{2}-1\right) \kappa^{4}=0 .
$$

Solving ODE (57), we have $\kappa=4 c_{2} / 16\left(c^{2}-1\right)+c_{2}^{2} s^{2}+2 c_{2}^{2} c_{3} s+\left(c_{2} c_{3}\right)^{2}$.

Conversely, making similar discussions as in Case 2, we prove that $\gamma$ is $f$-biharmonic.

Proof of Theorem 2. According to (21), we have

$$
\begin{aligned}
\nabla_{\gamma^{\prime}}^{\mathbb{L}^{3}} \widetilde{\mathbf{p}}_{1} & =\widetilde{\mathbf{p}}_{2}, \\
\nabla_{\gamma^{\prime}}^{\mathbb{L}^{3}} \nabla_{\gamma^{\prime}}^{\mathbb{L}^{3}} \gamma^{\prime} & =\widetilde{\tau} \widetilde{\mathbf{p}}_{2}, \\
\nabla_{\gamma^{\prime}}^{\mathbb{L}^{3}} \nabla_{\gamma^{\prime}}^{\mathbb{L}^{3}} \nabla_{\gamma^{\prime}}^{\mathbb{L}^{3}} \gamma^{\prime} & =\left(\widetilde{\tau}^{\prime}+\widetilde{\tau}^{2}\right) \widetilde{\mathbf{p}}_{2} .
\end{aligned}
$$

Using Lemma 2, we obtain that $\gamma$ is $f$-biharmonic if and only if

$$
f^{\prime \prime}+2 \widetilde{\tau} f \prime+\left(\tilde{\tau}^{\prime}+\tilde{\tau}^{2}\right) f=0
$$

(i) When $\tilde{\tau}$ is zero, then it follows from (59) that $f^{\prime \prime}=0$, which means that $f=c_{1} s+c_{2}$, where $c_{1}$ and $c_{2}$ are two constants. Meanwhile, we conclude from Theorem 3.5 in [32] that

$$
\gamma(s)=\left(\frac{s^{2}}{2}, s, \frac{s^{2}}{2}\right),
$$

which is a planar curve.

(ii) When $\tilde{\tau}$ is a nonzero constant, then (59) is equivalent to

$$
f^{\prime \prime}+2 \widetilde{\tau} f^{\prime}+\tilde{\tau}^{2} f=0
$$

Solving the above ODE, we obtain that $f=\left(c_{1}+c_{2} s\right) e^{-\tau s}$, where $c_{1}$ and $c_{2}$ are two constants, and know from Theorem 3.5 in [32] that

$$
\gamma=\frac{1}{\tilde{\tau}^{2}}\left(\cosh (\tilde{\tau} s)+\sinh (\tilde{\tau} s), \tilde{\tau}^{2} s, \cosh (\tilde{\tau} s)+\sinh (\tilde{\tau} s)\right),
$$

which is a helix curve (cf. [28]).

(iii) When $\tilde{\tau} \neq$ constant, then by solving ODE (59), we get that $f=\left(c_{1}+c_{2} s\right) e^{-\int \tilde{\tau} \mathrm{d} s}$, where $c_{1}$ and $c_{2}$ are two constants. Also, we know from [28] that $\gamma$ is a helix.

The converse is clear from the similar proof of Theorem 3.5 in [32], together with the corresponding function $f$.

As a consequence of Theorems 1 and 2, we obtain the following.

Corollary 1. Any $f$-biharmonic unit speed curve with arclength parameter in Lorentz-Minkowski 3-space is a helix.

Using (28), we obtain that $\gamma$ is biharmonic if and only if

$$
\gamma^{(\mathrm{iv})}=0 \text {. }
$$

According to (22), it is easy to find that when $f$ is a constant, $f$-biharmonic curves must be biharmonic ones. It is a natural and interesting problem: whether fmust be a constant when $f$-biharmonic curves are biharmonic. Unfortunately, this problem is not true.

For the $f$-biharmonic curve $\gamma$ in Theorem 2,

$$
\begin{aligned}
& \gamma=\left(\frac{s^{2}}{2}, s, \frac{s^{2}}{2}\right), \\
& f=c_{1} s+c_{2},
\end{aligned}
$$

where $c_{1}$ and $c_{2}$ are two constant. Using (63), it easily check that $\gamma$ is biharmonic, but $f$ is a function.

Remark 2. As we all know, the theorem of existence and uniqueness for curves in $\mathbb{R}^{3}$ asserts that given two functions $\kappa>0$ and $\tau$, there exists a unique (up a rigid motion) curve in $\mathbb{R}^{3}$ with the curvature $\kappa$ and the torsion $\tau$. In LorentzMinkowski space $\mathbb{L}^{3}$, the result of existence for curves is the same as that of in $\mathbb{R}^{3}$ (cf. [28], Thereoms 2.6-2.7). In general, the uniqueness for curves is not true by the causal character of the curve (cf. [28] for details). However, it holds if the causal character of the Frenet frame field agree for both curves $\gamma_{1}$ and $\gamma_{2}$ (i.e., the tangent, principal normal, and binormal of $\gamma_{1}$ have the same causal character compared to 
the corresponding tangent, principal normal, and binormal of $\gamma_{2}$, respectively) [33]. This, together with our classification theorems, implies that there are many examples of proper $f$-biharmonic curves in $\mathbb{L}^{3}$.

Finally, we give an example of a nonbiharmonic $f$-biharmonic curve in $\mathbb{L}^{3}$. For the $f$-biharmonic curve in Theorem 2,

$$
\gamma=\frac{1}{\tilde{\tau}^{2}}\left(\cosh (\widetilde{\tau} s)+\sinh (\widetilde{\tau} s), \tilde{\tau}^{2} s, \cosh (\widetilde{\tau} s)+\sinh (\widetilde{\tau} s)\right),
$$

and $f=\left(c_{1}+c_{2} s\right) e^{-\tilde{\tau} s}$ with $\tilde{\tau}$ be a nonzero constant.

Using (63), a short computation proves that $\gamma$ is a nonbiharmonic curve.

\section{Data Availability}

No data were used to support this study.

\section{Conflicts of Interest}

The authors declare that they have no conflicts of interest.

\section{Acknowledgments}

This work was supported by the Natural Science Foundation of Chongqing (No. cstc2019jcyj-msxmX0172); the Scientific and Technological Research Program of Chongqing $\mathrm{Mu}-$ nicipal Education Commission (No. KJQN201901128); the Scientific Research Starting Foundation of Chongqing University of Technology (No. 2017ZD52).

\section{References}

[1] J. Eells and L. Lemaire, "Selected topics in harmonic maps, CBMS regional conference series in mathematics," Journal of the American Mathematical Society, vol. 50, 1983.

[2] R. Caddeo, S. Montaldo, and C. Oniciuc, "Biharmonic submanifolds of $\mathrm{S}^{3}$," International Journal of Mathematics, vol. 12 , no. 8, pp. 867-876, 2001.

[3] T. Sasahara, "Biharmonic submanifolds in nonflat Lorentz 3space forms," Bulletin of the Australian Mathematical Society, vol. 85, no. 3, pp. 422-432, 2012.

[4] G.-Y. Jiang, "2-harmonic maps and their first and second variational formulas," Chinese Annals of Mathematics, Series A, vol. 7, no. 4, pp. 389-402, 1986.

[5] G.-Y. Jiang, "2-harmonic isometric immersions between Riemannian manifolds," Chinese Annals of Mathematics, Series A, vol. 7, no. 2, pp. 130-144, 1986.

[6] W. Lu, "On $f$-biharmonic maps and bi $f$-harmonic maps between Riemannian manifolds," Science China Mathematics, vol. 58, no. 7, pp. 1483-1498, 2015.

[7] S. Ouakkas, R. Nasri, and M. Djaa, "On the $f$-harmonic and $f$-biharmonic maps," JP Journal of Geometry and Topology, vol. 10, pp. 11-27, 2010.

[8] Y.-L. Ou, "Onf-biharmonic maps and f-biharmonic submanifolds," Pacific Journal of Mathematics, vol. 271, no. 2, pp. 461-477, 2014.

[9] B.-Y. Chen, "Some open problems and conjectures on submanifolds of finite type," Soochow Journal of Mathematics, vol. 17, pp. 169-188, 1991.
[10] B.-Y. Chen, "Surfaces with parallel normalized mean curvature vector," Monatshefte für Mathematik, vol. 90, no. 3, pp. 185-194, 1980.

[11] I. Dimitríc, "Submanifolds of $\mathbb{E}^{m}$ with harmonic mean curvature vector," Bulletin of the Institute of Mathematics, Academia Sinica, vol. 20, pp. 53-65, 1992.

[12] B.-Y. Chen and S. Ishikawa, "Biharmonic surfaces in pseudoEuclidean spaces," Memoirs of the Faculty of Science, Kyushu University, Series A, Mathematics, vol. 45, no. 2, pp. 323-347, 1991.

[13] R. Caddeo, S. Montaldo, and P. Piu, "Biharmonic curves on a surface," Rendiconti di Matematica e delle sue Applicazioni, vol. 21, pp. 143-157, 2001.

[14] B.-Y. Chen and S. Ishikawa, "Biharmonic pseudo-Riemannian submanifolds in pseudo-Euclidean spaces," Kyushu Journal of Mathematics, vol. 52, no. 1, pp. 167-185, 1998.

[15] I. Inouguchi, "Biharmonic curves in Minkowski 3-space," International Journal of Mathematics and Mathematical Sciences, vol. 21, pp. 1365-1368, 2003.

[16] R. Caddeo, S. Montaldo, C. Oniciuc, and P. Piu, "The classification of biharmonic curves of Cartan-Vranceanu 3-dimensional spaces," in Proceedings of the 7th International Workshop on Differential Geometry and its Applications, ClujNapoca, Romania, 2006.

[17] R. Caddeo, C. Oniciuc, and P. Piu, "Explicit formulas for nongeodesic biharmonic curves of the Heisenberg group," Rendiconti del Seminario Matematico Università e Politecnico di Torino, vol. 62, pp. 265-277, 2004.

[18] S. Montaldo and A. Ratto, "Biharmonic curves into quadrics," Glasgow Mathematical Journal, vol. 57, no. 1, pp. 131-141, 2015.

[19] N. Voicu, "Biharmonic curves in Finsler spaces," Journal of the Korean Mathematical Society, vol. 51, no. 6, pp. 1105-1122, 2014.

[20] B.-Y. Chen and M. I. Munteanu, "Biharmonic ideal hypersurfaces in Euclidean spaces," Differential Geometry and its Applications, vol. 31, no. 1, pp. 1-16, 2013.

[21] Y. Dong and Y.-L. Ou, "Biharmonic submanifolds of pseudoRiemannian manifolds," Journal of Geometry and Physics, vol. 112, pp. 252-262, 2017.

[22] L. Du and J. Zhang, "Biharmonic submanifolds with parallel normalized mean curvature vector field in pseudo-Riemannian space forms," Bulletin of the Malaysian Mathematical Sciences Society, vol. 42, no. 4, pp. 1469-1484, 2019.

[23] T. Liang and Y.-L. Ou, "Biharmonic hypersurfaces in a conformally flat space," Results in Mathematics, vol. 64, no. 12, pp. 91-104, 2013.

[24] N. Nakauchi and H. Urakawa, "Biharmonic submanifolds in a Riemannian manifold with non-positive curvature," Results in Mathematics, vol. 63, no. 1-2, pp. 467-474, 2013.

[25] Ş. Güvenç and Ö. ÖzgurCihan, "On the characterizations of $f$ biharmonic legendre curves in Sasakian space forms," Filomat, vol. 31, no. 3, pp. 639-648, 2017.

[26] F. Karaca and C. Özgür, "On $f$-biharmonic Curves," International Electronic Journal of Geometry, vol. 11, no. 2, pp. 18-27, 2018.

[27] J. Roth and A. Upadhyay, "f-biharmonic submanifolds of generalized space forms," Results in Mathematics, vol. 75, p. 20, 2020.

[28] R. López, "Differential geometry of curves and surfaces in Lorentz-Minkowski space," International Electronic Journal of Geometry, vol. 7, no. 1, pp. 44-107, 2014.

[29] A. Ferrández, P. Lucas, and M. A. Meroño, "Biharmonic Hopf cylinders," Rocky Mountain Journal of Mathematics, vol. 28, no. 3, pp. 957-975, 1998. 
[30] S. Izumiya and A. Takiyama, "A time-like surface in Minkowski 3-space which contains pseudocircles," Proceedings of the Edinburgh Mathematical Society, vol. 40, no. 1, pp. 127136, 1997.

[31] S. Izumiya and A. Takiyama, "A time-like surface in Minkowski 3-space which contains light-like lines," Journal of Geometry, vol. 64, no. 1-2, pp. 95-101, 1999.

[32] J. Walrave, Curves and surfaces in Minkowski space, Ph.D. thesis, K. U. Leuven, Fac. of Science, Leuven, 1995.

[33] T. Ikawa, "On curves and submanifolds in an indefiniteRiemannian manifold," Tsukuba Journal of Mathematics, vol. 9, no. 2, pp. 353-371, 1985. 